\title{
Effect of inelastic scattering on parametric pumping
}

\author{
M. Moskalets ${ }^{1,2}$ and M. Buttiker ${ }^{1}$ \\ ${ }^{1}$ Département de Physique Théorique, Université de Genève, CH-1211 Genève 4, Switzerland \\ ${ }^{2}$ Department of Metal and Semiconductor Physics, National Technic University "Kharkov Polytechnic Institute", Kharkov, \\ Ukraine
}

(February 7, 2020)

\begin{abstract}
Pumping of charge in phase-coherent mesoscopic systems due to the out-of-phase modulation of two parameters has recently found considerable interest. We investigate the effect of inelastic processes on the adiabatically pumped current through a two terminal mesoscopic sample. We find that the loss of coherence does not suppress the pumped charge but rather an additional physical mechanism for an incoherent pump effect comes into play. In a fully phase incoherent system the pump effect is similar to a rectification effect.
\end{abstract}

PACS numbers: 72.10.-d, 73.23.-b

A recent experiment by Switkes et al.1 generated considerable interest in the adiabatic quantum pumping of charge. In this experiment a dc voltage between two electron reservoirs of an open quantum dot was obtained by slow periodic but out-of-phase variation of two independent parameters. The dc potential difference is a consequence of electron transfer between reservoirs. The effect is discussed under conditions in which electron motion is completely phase-coherent and is therefore termed quantum pumping. Here we are interested in the effect of inelastic scattering. Interestingly, as we will show inelastic scattering does not necessarily suppress the pumped charge but can even enhance it.

There are several approaches to achieve charge pumping. Perhaps the most direct way to generate charge transport without applying an overall force to the system is to subject the system to a traveling wave potential as envisaged by Thoulesst. In that case there are conditions under which the pumped charge is quantized 3 , 3 . The purely classical limit is also intersting and has been used as a model for a clocked Brownian computelt. Travelling potentials can be generated for instance with the help of surface acoustic wave 3 . Ansther possibility is to exploit the Coulomb blockade effect 9 that leads to a quantization of acharge on a quantum dot and is of metrological interest 10 . Still another possibility 122 22, called an adiabatic quantum electron pump, is based on an open dot under conditions in which Coulomb blockade effects are not important. In this case the pumped charge (either quantized or nonquantized) depends on the interference of electron wave functions within the system. It is this case which is of interest in this work. Inelastic scattering destroies interference and we might naively expect that the pump effect is suppressed as inelastic scattering increases.

An elegant formulation of the charge pumped through a phase coherent conductor coupled to two reservoirs with the same (electro-) chemical potentials $\mu_{l}=\mu_{r}=\mu$ (see Fig.1) has been given by Brouwer 13 . He derived a scattering matrix expression for the pumped charge in- vestigating the modulation of the charge emissivity 2324. If some two parameters $X_{1}, X_{2}$ characterizing the system are varied adiabatically and periodically (with the frequency $\omega=2 \pi / \tau \rightarrow 0$ ) the charge $Q$ transferred durino one period from the right reservoir to the left one is 13

$$
Q=e \int_{0}^{\tau} d t\left(\frac{d n_{l}}{d X_{1}} \frac{d X_{1}}{d t}+\frac{d n_{l}}{d X_{2}} \frac{d X_{2}}{d t}\right) .
$$

Here $d n_{l} / d X$ is the emitance 23,24 into contact (lead) "l"

$$
\frac{d n_{\alpha}}{d X}=\frac{1}{2 \pi} \sum_{\beta=l, r} \operatorname{Im}\left[\frac{\partial S_{\alpha \beta}}{\partial X} S_{\alpha \beta}^{*}\right],
$$

where $S_{\alpha \beta}(\alpha, \beta=l, r)$ are elements of a scattering matrix of a system under consideration (note that because of a current conservation $Q$ may be expressed through $-d n_{r} / d X$ in the same way).

The physical mechanism for adiabatic (parametric) pumping is as follows. The infinitesimal change of system parameters $\delta X_{i}$ (for a time $\delta t$ ) leads to a redistribution of the charge within (and around) the system. The redistribution of the charge is a consequence of the variation of the electron density of states and produces electron flows through the system 13 where $\delta Q_{i}\left(X_{1}, X_{2}\right)=e d n / d X_{i} \delta X_{i},(i=1,2)$. These currents (and the pumping effect) are thus a consequence of the changing electrostatic landscape.

The effect of inelastic interactions may be understood in the following way. To be definite let us consider a quantum dot coupled to reservoirs via two point contacts. Without inelastic interactions electrons pass coherently from one reservoir to another and the pumped charge shows a resonant like behavior 17128 that is a consequence of interference between the two point contacts. The inelastic interactions within a quantum dot destroy this quantum interference and allow us to consider the dot's interior as a third reservoir 25 with some potential $\mu_{f}(t)$ which is determined such that the net current into this additional reservoir is zero at every instant. 


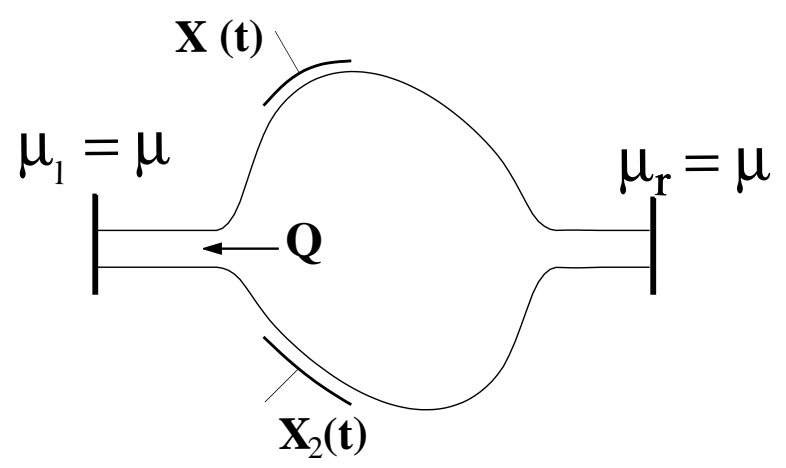

FIG. 1. A mesoscopic system coupled to two reservoirs with the same potentials $\mu_{l}=\mu_{r}=\mu$. When the parameters $X_{1}$ and $X_{2}$ are changed periodically but out phase a charge $Q$ can be transferred from one reservoir to another one.

For a typical geometry the currents pumped through the two contacts are unequal and some charge is pumped to the third reservoir generating a time-dependent potential $\mu_{f}(t)$. Due to this potential additional currents flow into (or from) the reservoirs. The existence of these additional currents is at the origin of an inelastically driven electron pump. Thus in the presence of inelastic interactions there is a novel rectification mechanism which as we will show can give rise to a dc current. We emphasize already here, that this effect differs from the rectification of displacement currents recently discussed by Brouwer 20 and Polianski and Brouwer 21 which is closely relatef to the actual set-up of the experiment of Switkes et al.t.

To illustrate the inelastic rectification mechanism giving raise to a pumping effect we consider a generic case: a point scatterer with an oscillating strength coupled to two reservoirs with a periodic in time chemical potential difference $\delta \mu(t)=\mu_{l}-\mu_{r}$. The time-modulation of the point scatterer leads to a conductance

$$
G(t)=G_{0}+\delta G \sin (\omega t)
$$

and the chemical potential difference is

$$
\delta \mu(t)=\delta \mu \sin (\omega t+\varphi)
$$

with $\varphi$ an arbitrary phase.

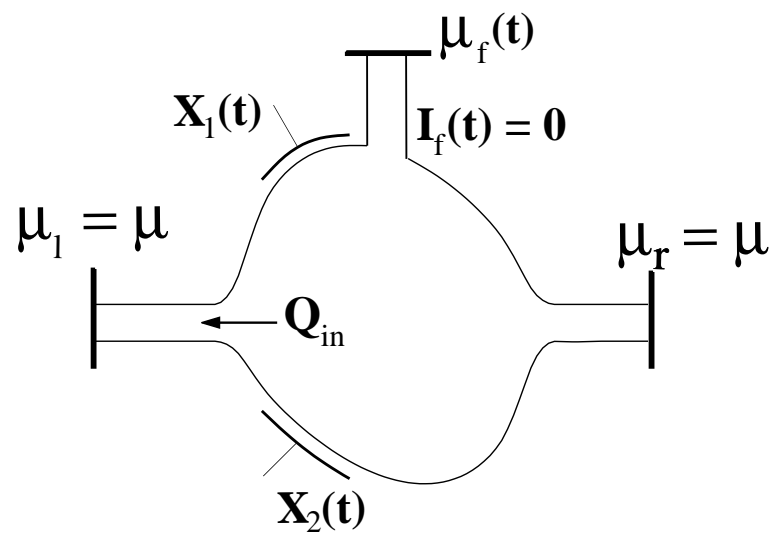

FIG. 2. To introduce inelastic interactions we couple the system (Fig.1) to the third (fictitious) reservoir with a time-dependent chemical potential $\mu_{f}(t)$. The net current into this reservoir is zero at any time $I_{f}(t)=0$.

The charge $Q$ transferred through the sample in one period $\tau=2 \pi / \omega$ is $Q=\int_{0}^{\tau} d t G(t) \delta \mu(t)$. Using Eqs.(3), (4) we find a dc current $I_{d c}=Q / \tau$ pumped through the sample given by

$$
I_{d c}=\frac{1}{2} \delta G \delta \mu \cos (\varphi)
$$

Note, that this pump driven by rectification has similar features as the quantum coherent adiabatic pump. Namely, this effect exists if at least two system parameters are varied in time. In the above example these parameters are the point scatterer (the conductance) and the inelastically generated potential difference. In addition, the pumped current $I_{d c}$ given by Eq.(5) is proportional to the frequency $\omega$. This follows from the fact (see the discussion after Eq.(7) that in the adiabatic pump considered here the potential difference (produced by inelastic interactions) $\delta \mu \sim I_{i} \sim d X_{i} / d t \sim \omega$ is also proportional to the pump frequency $\omega$. So, we have $I_{d c} \sim \omega$ as it must be for a pump effect.

Below we present a more formal derivation which allows us to consider the effect of inelastic interactions on the pump effect within a scattering approach. To introduce inelastic interactions into the system under consideration we will use the model of Ref. 25 in which a third, fictitious voltage probe is coupled to the conductor (see Fig.2). We can only give a few recent references to indicate the breadth of questions addressed with this approach26. The net current $I_{f}(t)$ between the system and this additional reservoir is taken to be zero at any time (i.e., the charge leaving the system through the third lead for the time $\delta t$ is zero, $\left.\delta Q_{f}(t)=I_{f}(t) \delta t=0\right)$. Note, that this assumption leads to a time dependent electrochemical potential difference between the fictitious reservoir and the system (and, respectively, between fictitious and real reservoirs). Some carriers are scattered into the fictitious reservoir and the resulting current is compensated by carriers injected from the reservoir into the sys- 
tem. Carriers entering and leaving a reservoir have a phase and energy which is unrelated. Physically the third reservoir describes thus carriers which undergo an inelastic scattering process within the mesoscopic sample and as a consequence loose their phase coherence.

The presence of the third lead modifies the charge $Q_{\alpha}$ leaving the sample through the leads $\alpha=l, r$. After straightforward calculations taking into account that $\delta Q_{f}(t)=0$ we get a pumped charge $Q_{i n}$ due to inelastic interactions of the following form

$$
\begin{aligned}
Q_{i n} & =Q_{i n, l}=-Q_{i n, r} ; \\
Q_{i n, \alpha} & =e \int_{0}^{\tau} d t\left(F(\alpha)+K_{i n, \alpha} F(f)\right), \quad \alpha=l, r, \\
F(\gamma) & =\frac{d n_{i n, \gamma}}{d X_{1}} \frac{d X_{1}}{d t}+\frac{d n_{i n, \gamma}}{d X_{2}} \frac{d X_{2}}{d t}, \quad \gamma=l, r, f .
\end{aligned}
$$

Here the emissivities $d n_{i n, \gamma} / d X(\gamma=l, r, f)$ are

$$
\frac{d n_{i n, \gamma}}{d X}=\frac{1}{2 \pi} \sum_{\beta=l, r, f} \operatorname{Im}\left[\frac{\partial S_{\gamma \beta}}{\partial X} S_{\gamma \beta}^{*}\right] .
$$

In Eq. (7) the index of summation now also runs over the fictitious lead. The coefficients $K_{i n, \alpha}=\frac{T_{f \alpha}}{T_{f l}+T_{f r}}$ (where $T_{f \alpha}=\left|S_{f \alpha}\right|^{2}$ is the probability for electrons entering the sample through the lead $\alpha=l, r$ to be inelastically scattered within the sample) describe a redistribution of outgoing particles undergoing inelastic processes within the system between the left and the right leads.

In the above expression the second term contains a new (compared with a purely coherent case) physical mechanism for an adiabatic electron pump. This mechanism is a rectification of incoherent currents flowing into the system from the left and the right reservoirs (or vice versa). When the system parameters $X_{1}$ and $X_{2}$ are varied the current flowing into the third (fictitious) reservoir (i.e., an incoherent current) is $I_{i n}(t)=e F(f)$. To avoid a charge accumulation within the system (within the third reservoir) this current induces an additional potential $\delta \mu(t)=\mu_{f}(t)-\mu=I_{\text {in }}(t) / G\left(\right.$ where $\left.G=e^{2} / h\left(T_{f l}+T_{f r}\right)\right)$ in the system interior in order to maintain the full current $I_{f}$ into this reservoir at zero. The presence of a potential $\delta \mu(t)$ changes the currents flowing into the real reservoirs by amount $\Delta I_{\alpha}(t)=e^{2} / h T_{f \alpha} \delta \mu=I_{\text {in }}(t) K_{\text {in } \alpha}(t)$. Integrating $\Delta I_{\alpha}$ over a time period $\tau$ we get an additional charge pumped through the system. This charge has a rectified part that survives even if the coherent pump effect is fully destroyed by inelastic interactions.

To provide an example we now consider the simplest system which can pump a charge. As was shown by Brouwer 3 parametric pumping may occur if at least two (independent) system parameters are varied. From this it immediately follows that a point scatterer (a delta function potential) with a potential $U_{1}(x, t)=V(t) \delta(x)$ does not show a pump effect, because in this case the scattering is characterized by one parameter only - the strength $V$. Thus, only a partially extended system can pump a charge. Thus we consider two point scatterers located at some distance from each other $U(x, t)=V_{l}(t) \delta(x+a)+$ $V_{r}(t) \delta(x-a)$. The pump effect in such a coherent system was considered in 17.18 . Inelastic interactions destroy coherent tunneling and lead to a sequential (incoherent) tunneling through two barriers25.

To introduce inelastic interactions we couple the system to the third reservoir using a wave-splitter located at $x=0$ described by a scattering matrix $S_{e}{ }^{2}$

$$
S_{e}=\left(\begin{array}{cccc}
0 & \sqrt{1-\epsilon} & \sqrt{\epsilon} & 0 \\
\sqrt{1-\epsilon} & 0 & 0 & \sqrt{\epsilon} \\
\sqrt{\epsilon} & 0 & 0 & -\sqrt{1-\epsilon} \\
0 & \sqrt{\epsilon} & -\sqrt{1-\epsilon} & 0
\end{array}\right)
$$

Here the coupling parameter $\epsilon$ characterizes the strength of inelastic interactions. At $\epsilon=1$ all electrons are inelastically scattered within the system. Whereas at $\epsilon=0$ electrons move coherently through the system (from channel 1 to channel 2) and a fictitious reservoir (channels 3 and 4) fully decoupled from the system under consideration.

First, we consider the limit of strong inelastic interactions $\epsilon=1$. In this case we have no coherent tunneling 25 and the system may be considered as two classical resistors in series (point potential barriers with heights $\left.V_{\alpha}(t), \alpha=l, r\right)$ with the scattering matrices $S_{11}^{(\alpha)}=S_{22}^{(\alpha)}=-i \xi_{\alpha} /\left(1+i \xi_{\alpha}\right) ; \quad S_{12}^{(\alpha)}=S_{21}^{(\alpha)}=1 /\left(1+i \xi_{\alpha}\right)$, where $\xi_{\alpha}=V_{\alpha} /(\hbar v)$ and $v$ is an electron velocity. To avoid a misunderstanding note, that at $\epsilon=1$ all electrons entering the system through the channel 1 (2) go into channel 3 (4) (and vice versa). Thus, the system containing four leads (two real channels 1 and 2 and two fictitious channels 3 and 4) is divided into two subsystems (the scatterers $V_{l}$ and $V_{r}$ ) with two leads only (channels

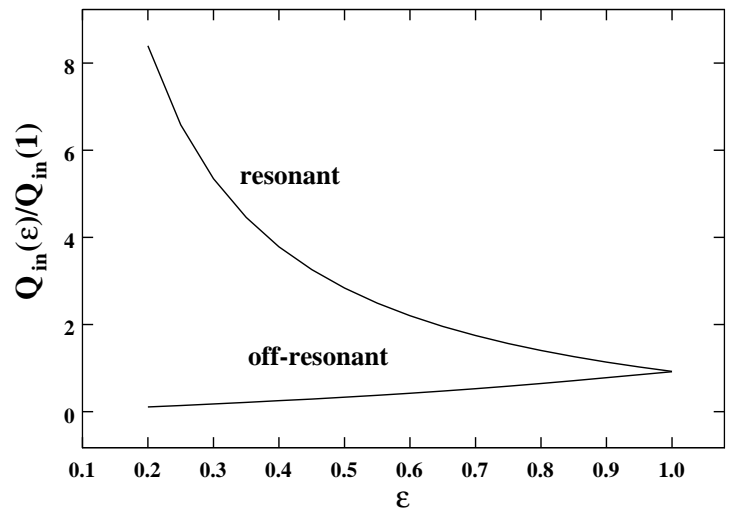

FIG. 3. The dependence on the strength $\epsilon$ of inelastic interactions of the charge $Q_{\text {in }}$ pumped through a double-barrier potential $U(x)=V_{l}(t) \delta(x+d)+V_{r}(t) \delta(x-d)$ at small variations of the potential strengths $V_{\alpha}(t)=V_{0}+\delta V_{\alpha}(t), \delta V_{\alpha} \ll V_{0}$, $\alpha=l, r$. The parameters are: $V_{0} /(\hbar v)=5 ; k_{F} d=3.04$ modulo $\pi$ (resonant); $k_{F} d=\pi / 4$ modulo $\pi$ (off-resonant). Here $v, k_{F}$ are the electron velocity and Fermi wave vector. 
$(1,3)$ and $(2,4)$, respectively). Though each of the subsystems has only one parameter $\left(V_{\alpha}(t)\right)$ which is varied (and, consequently, they do not show a coherent pump effect) the full system can pump current due to a rectification mechanism. Substituting $K_{i n, l}=G_{l} /\left(G_{l}+G_{r}\right)$ and the scattering matrix $S^{\alpha}$ into Eqs.(6) and (7) we get a pumped charge

$$
Q_{i n}=-\frac{1}{e v} \int_{0}^{\tau} d t\left(G_{l}^{-1}(t)+G_{r}^{-1}(t)\right)^{-1}\left\{\frac{\partial V_{l}}{\partial t}-\frac{\partial V_{r}}{\partial t}\right\} .
$$

Here $G_{\alpha}=\left(e^{2} / h\right)\left|S_{12}^{\alpha}\right|^{2}$.

In the limit of a small modulation of the height of the potential barriers $V_{\alpha}(t)=V_{0 \alpha}+\delta V_{\alpha} \sin \left(\omega t+\varphi_{\alpha}\right), \delta V_{\alpha} \ll$ $V_{0 \alpha}(\alpha=l, r)$ for equal opaque barriers $V_{0 l}=V_{0 r}=V_{0}$ and $V_{0} \gg \hbar v$ we get a charge pumped for one period $\tau$

$$
Q_{i n}=e \sqrt{T_{0}} \frac{\delta G_{l} \delta G_{r}}{8 G_{0}^{2}} \sin \left(\varphi_{r}-\varphi_{l}\right)
$$

where $G_{0}=e^{2} / h T_{0} ; \quad T_{0}=\left(\hbar v / V_{0}\right)^{2}$ and $\delta G_{\alpha}=-2 G_{0} \delta V_{\alpha} / V_{0}$.

Let us compare $Q_{i n}$ with the pumped charge 18 in the fully coherent limit. For a small variation $\delta V_{\alpha} \ll V_{0}$ the off-resonant pumped charge is $Q_{o f f} \sim$ $e T_{0} \sqrt{T_{0}} \delta G_{l} \delta G_{r} / G_{0}^{2}$. Thus away from a transmission resonance the inelastic interactions increase the pumped charge by a factor of $\sim 1 / T_{0}$.

Near a resonance it is more convenient to consider $a$ large pumped cycle which encloses a resonance line 18 . In this case the coherent pumped charge is quantized. The inelastic interactions destroy the Fabry-Perot interference effect and destroy the quantization of the pumped charge. In particular, for a limit cycle which encloses (counterclockwise) a region $0<V_{l}, V_{r}<\infty$ the equation (9) gives $Q_{\text {in }}=e / 2^{3 / 2}$.

These results are illustrated in Fig.3 which shows the effect of inelastic scattering away from resonance and on resonance as a function of the coupling strength $\epsilon$ to the fictitious voltage lead. Whereas near a resonance inelastic scattering suppresses the pumped charge (we assume that the potential variations do not lead us away from the resonant region, i. e. a small amplitude pump cycle) the off-resonant pump current is increased by inelastic scattering. Note, that the inelastic interactions affect the confyctance of a double barrier structure in an analogous way 25 .

In conclusion, we have shown that inelastic interactions within a mesoscopic system introduce an additional mechanism which contributes to adiabatic electron pumping.

M.M. appreciates the warm hospitality of the Department of Theoretical Physics of the University of Geneva, where part of this work was done. This work was supported by the Swiss National Science Foundation.
${ }^{1}$ M. Switkes, C.M. Marcus, K. Campman, A.C. Gossard, Science 283, 1905 (1999).

${ }^{2}$ D.J. Thouless, Phys. Rev. B 27, 6083 (1983).

${ }^{3}$ Q. Niu, Phys. Rev. Lett. 64, 1812 (1990).

${ }^{4}$ R. Landauer and M. Büttiker, Physica Scripta, T9, 155 (1985).

${ }^{5}$ V. I. Talyanskii, J. M. Shilton, M. Pepper, C. G. Smith, C. J. Ford, E. H. Linfield, D. A. Ritchie, and G. A. C. Jones, Phys. Rev. B 56, 15180 (1997).

${ }^{6}$ L.P. Kouwenhoven, A.T. Johnson, N.C. van der Vaart, C.J.P.M. Harmans, and C.T. Foxon, Phys. Rev. Lett 67, 1626 (1991).

${ }^{7}$ H. Pothier, P. Lafarge, C. Urbina, D. Esteve, and M.H. Devoret, Europhys. Lett. 17, 249 (1992).

8 T.H. Oosterkamp, L.P. Kouwenhoven, A.E.A. Koolen, N.C. van der Vaart, and C.J.P.M. Harmans, Phys. Rev. Lett. 78, 1536 (1997).

${ }^{9}$ B. L. Hazelzet, M. R. Wegewijs, T. H. Stoof, and Yu. V. Nazarov Physical Review B 63, 165313 (2001).

${ }^{10}$ M. Covington, Mark W. Keller, R. L. Kautz, and John M. Martinis Phys. Rev. Lett. 84, 5192 (2000).

${ }^{11}$ I.L. Aleiner, A.V. Andreev, Phys. Rev. Lett. 81, 1286 (1998).

12 B. Spivak, F. Zhou, M.T. Beal Monod, Phys. Rev. B 51, 13226 (1995).

${ }^{13}$ P.W.Brouwer, Phys. Rev. B 58, R10135 (1998).

${ }^{14}$ F. Zhou, B. Spivak, and B. Altshuler, Phys. Rev. Lett. 82, 608 (1999).

15 T.A. Shutenko, I.L. Aleiner, and B.L. Altshuler, Phys. Rev. B 61, 10366 (2000).

16 S. H. Simon, Phys. Rev. B 61, R16327 (2000).

17 Yadong Wei, Jian Wang, and Hong Guo, Phys. Rev. B 62, 9947 (2000).

${ }^{18}$ Y. Levinson, O. Entin-Wohlman, P. Wölfle, condmat/0010494 (2000).

${ }^{19}$ J.E. Avron, A. Elgart, G.M. Graf, and L. Sadun, Phys. Rev. B 62, R10618 (2000).

${ }^{20}$ P.W.Brouwer, Phys. Rev. B 63, 121303 (2001).

${ }^{21}$ M. L. Polianski and P.W.Brouwer, Phys. Rev. B 64, 075304 (2001)

22 J.E. Avron, A. Elgart, G.M. Graf and L. Sadun, mathph/0105011 (2001).

${ }^{23}$ M. Büttiker, H. Thomas, and A. Prétre, Z. Phys. B 94, 133 (1994).

${ }^{24}$ M. Büttiker, J. Phys. Condens. Matter 5, 9361 (1993).

${ }^{25}$ M. Büttiker, IBM J. Res. Develop. 32, 63 (1988); Phys. Rev. B 33, 3020 (1986).

${ }^{26}$ M.J.M. de Jong and C.W.J. Beenakker, Physica A 230, 219 (1996); N.A. Mortensen, A.-P. Jauho, K. Flensberg, and H. Schomerus, Phys. Rev. B 60, 13762 (1999); M.T. Liu and C.S. Chu, Phys. Rev. B 61, 7645 (2000); C. Texier and M. Büttiker, Phys. Rev. B 62, 7454 (2000); J. Shi and X.C. Xie, Phys. Rev. B 63, 045123 (2001); Xin-Qi Li, YiJing Yan, cond-mat/0106439 (2001). 